\title{
Natural phenolic derivatives based on piperine scaffold as potential antifungal agents
}

\author{
Jingjing Wang, Wenlong Wang, Haojin Xiong, Di Song and Xiufang Cao*
}

\begin{abstract}
Piperine is a natural alkaloid with a wide range of biological functions. Natural phenolic compounds existed in many essential oils (EOs) are plant-derived aroma compounds with broad range of biological activities, however, their actions are slow, and they are typically unstable to light or heat, difficult to extract and so on. In order to find highpotential fungicides derived from piperine, a series of piperine-directed essential oil derivatives were designed and synthesized. The structures of all molecules were confirmed by satisfied spectral data, including ${ }^{1} \mathrm{H} \mathrm{NMR},{ }^{13} \mathrm{C} \mathrm{NMR}$ and ESIMS. The target compounds were screened for their potential fungicidal activities against six species of plant pathogen fungi, including Rhizoctonia solani, Fusarium graminearum, Phomopsis adianticola, Alternaria tenuis Nees, Phytophthora capsici and Gloeosporium theae-sinensis. Some of target compounds exhibited moderate and broad-spectrum activity against tested fungi compared to the parental piperine. Further studies have shown that some different concentrations of compounds have significant inhibitory activity against Alternaria tenuis Nees and Phytophthora capsici compared to commercial carbendazim, and compound $\mathbf{2} \mathbf{b}$ exhibited particularly significant broad-spectrum fungicidal activity.
\end{abstract}

Keywords: Piperine, Essential oils, Synthesis, Fungicidal activity

\section{Introduction}

Piperine, a natural amide compound, is the main active substance extracted of Piper nigrum Linn. As an important natural alkaloid, piperine exhibited a wide spectrum of biological and pharmacological activities [1-6], it has anti-oxidation, antidepressant [7], toxic effect against hepatocytes [8], antiapoptotic efficacy [9], high immunomodulatory and antitumor activity [4], and has obvious effects in lowering blood fat [10]. Clinically, it can effectively control the incidence of hyperlipidemia, the treatment rate is as high as $93.3 \%$, and it can also reduce the incidence of cardiovascular and cerebrovascular diseases. In addition to being used as a medicine, piperine is also an important organic synthetic building blocks

\footnotetext{
*Correspondence: caoxiufang@mail.hzau.edu.cn

College of Science, Huazhong Agricultural University, Wuhan 430070, China
}

and intermediate [11]. Its structure is mainly divided into three parts: piperidine ring, aromatic heterocyclic ring, and aliphatic hydrocarbon chain. These three places are usually considered by the researchers to be essential for their biological activity, and by modifying the structure of these parts, the biological activity of the compounds can be changed.

Essential oils (EOs) are class of complex mixtures of low molecular weight compounds extracted from various plants by steam distillation and various solvents [12]. Plant essential oils have received extensive attention from plant protection experts in recent years due to their low toxicity to mammals, low residue and extensive biological activity [13-15]. At present, there are many varieties of plant essential oils, and their applications are limited to the contact, fumigation and repellent of pests in confined environments such as greenhouses and warehouses [16-18]. In addition, essential oils can also be used as 
synergists, solubilizers, flavoring agents and chemical pesticides. However, most of essential oils are volatile, unstable to light and heat, easy to decompose, etc. Therefore, if the rational derivatization of essential oil molecules can be based on retaining their activity, the application of plant essential oils will undoubtedly be a significant development. Recently, during the course of our research for functional molecules based on natural essential oils $[19,20]$, a series of essential oil-oriented derivatives have been synthesized and approved to exhibit insecticidal or fungicidal activities, which suggest that these natural essential oils might contribute to the biological functions.

Based on this investigation, a series of piperine-oriented derivatives derived from natural phenolic compounds existed in essential oils were designed and synthesized as following strategy in Fig. 1. So, in order to explore the potential applications for these novel essential oil derivatives, we report herein the synthesis and characterization of twenty-one essential oil derivatives via simple reaction, and their antifungal activities against several phytopathogenic fungi have also been fully investigated.

\section{Materials and methods}

\section{Instrumentation and chemicals}

All chemicals or reagents used for syntheses were of analytical reagent, and used directly without purification. Melting points (m.p.) were determined on a RY-2 apparatus and are uncorrected. ${ }^{1} \mathrm{H}$ NMR spectra were recorded on a Brucker spectrometer at $600 \mathrm{MHz}$ with the $\mathrm{CDCl}_{3}$ as the solvent and TMS as the internal standard. ${ }^{13} \mathrm{C}$ NMR spectra were recorded on a Brucker spectrometer at $150 \mathrm{MHz}$ with $\mathrm{CDCl}_{3}$ as the solvent. Mass spectra were performed on a Waters ACQUITY UPLC ${ }^{\circledR}$ H-CLASS PDA (Waters ${ }^{\circledR}$ ) instrument. Column chromatography was carried out using silica gel 100-200 mesh. Analytical thin-layer chromatography (TLC) was carried out on precoated plates, and spots were visualized with ultraviolet light.

\section{General synthesis of precursors}

The key precursors including $(E)$-3-(benzo[d][1,3]dioxol5 -yl)acrylic acid $(\mathrm{n}=1)$ and piperic acid $(\mathrm{n}=2)$ were prepared using a similar methods reported in the references $[21,22]$.

\section{General synthetic procedures for target compounds}

The corresponding acid bearing 1,3-benzodioxole unit $(0.005 \mathrm{~mol})$, phenolic compound $(0.005 \mathrm{~mol})$ and acetonitrile $(30-60 \mathrm{~mL})$ were added to a $150 \mathrm{~mL}$ dry round bottom flask, and $0.3 \mathrm{~g}$ of 4-dimethylaminopyridine was added as a catalyst, and $1.5 \mathrm{~g}$ of $N, N^{\prime}$ dicyclohexylcarbodiimide was further added as a condensing agent. The reaction was stirred at room temperature to $40{ }^{\circ} \mathrm{C}$ for additional hours, and TLC traced the reaction to completion. After the completion of the reaction, the solution was dissolved in water $(20 \mathrm{~mL})$, and the aqueous solution was extracted with ethyl acetate $(30 \mathrm{~mL} \times 2)$ twice. The combined organic phases were washed with $5 \% \mathrm{Na}_{2} \mathrm{CO}_{3}$ solution $(30 \mathrm{~mL} \times 2)$ and water to neutrality and dried over anhydrous $\mathrm{Na}_{2} \mathrm{SO}_{4}$. After filtration and concentration, the corresponding crude compound were obtained, which were purified by silica gel column-chromatography (ethyl acetate/petroleum ether) or recrystallization to give pure compounds.

\section{5-Isopropyl-2-methylphenyl benzo[d][1,3] \\ dioxole-5-carboxylate (1a)}

${ }^{1} \mathrm{H}$ NMR $\left(600 \mathrm{MHz}, \mathrm{CDCl}_{3}\right): \delta=7.85(\mathrm{dd}, J=8.2,1.8 \mathrm{~Hz}$, $1 \mathrm{H}), 7.64(\mathrm{~d}, J=1.8 \mathrm{~Hz}, 1 \mathrm{H}), 7.19(\mathrm{~d}, J=7.8 \mathrm{~Hz}, 1 \mathrm{H}), 7.05$ $(\mathrm{dd}, J=7.8,1.8 \mathrm{~Hz}, 1 \mathrm{H}), 6.98(\mathrm{~d}, J=1.8 \mathrm{~Hz}, 1 \mathrm{H}), 6.92(\mathrm{~d}$, $J=8.2 \mathrm{~Hz}, 1 \mathrm{H}), 6.08(\mathrm{~s}, 2 \mathrm{H}), 2.93-2.88(\mathrm{~m}, 1 \mathrm{H}), 2.18$ (s, $3 \mathrm{H}), 1.25(\mathrm{~d}, J=6.6 \mathrm{~Hz}, 6 \mathrm{H}) ;{ }^{13} \mathrm{C} \mathrm{NMR}\left(150 \mathrm{MHz}, \mathrm{CDCl}_{3}\right)$ : $\delta=164.39,152.25,149.59,148.23,148.04,131.03,127.51$, 126.26, 124.26, 123.63, 120.04, 110.08, 108.31, 102.08, 33.74, 24.07, 15.98; MS (ESI) $m / z 299.6(\mathrm{M}+\mathrm{H})^{+}$, calcd. for $\mathrm{C}_{18} \mathrm{H}_{19} \mathrm{O}_{4} m / z=299.1$.

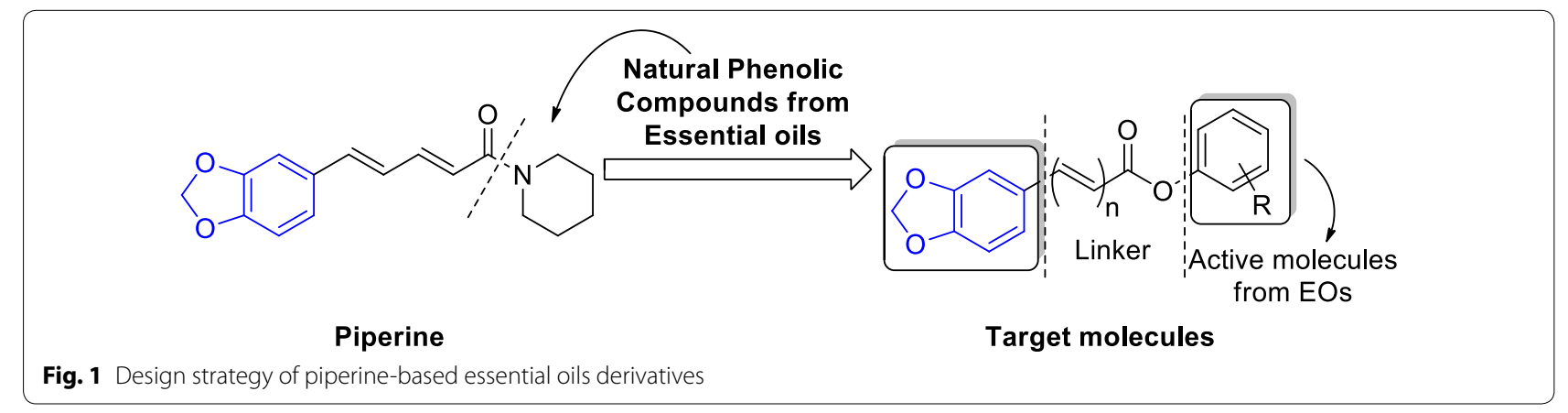




\section{2-Isopropyl-5-methylphenyl benzo[d][1,3] dioxole-5-carboxylate (1b)}

${ }^{1} \mathrm{H}$ NMR $\left(600 \mathrm{MHz}, \mathrm{CDCl}_{3}\right): \delta=7.84-7.82(\mathrm{~m}, 1 \mathrm{H}), 7.62$ (s, 1H), 7.26-7.20 (m, 1H), $7.05(\mathrm{~d}, J=7.4 \mathrm{~Hz}, 1 \mathrm{H}), 6.94-$ $6.88(\mathrm{~m}, 3 \mathrm{H}), 6.06(\mathrm{~s}, 2 \mathrm{H}), 3.06-3.01(\mathrm{~m}, 1 \mathrm{H}), 2.33(\mathrm{~s}, 3 \mathrm{H})$, $1.20(\mathrm{~d}, J=7.2 \mathrm{~Hz}, 6 \mathrm{H}) ;{ }^{13} \mathrm{C} \mathrm{NMR}\left(150 \mathrm{MHz}, \mathrm{CDCl}_{3}\right)$ : $\delta=164.72,152.15,148.15,147.93,137.17,136.60,127.10$, $126.43,126.12,123.51,122.90,109.91,108.20,101.96$, 27.29, 22.67, 20.85; MS (ESI) $m / z 299.5(\mathrm{M}+\mathrm{H})^{+}$, calcd. for $\mathrm{C}_{18} \mathrm{H}_{19} \mathrm{O}_{4} m / z=299.1$.

\section{Benzo[d][1,3]dioxol-5-yl benzo[d][1,3]dioxole-5-carboxylate (1c)}

${ }^{1} \mathrm{H} \mathrm{NMR}\left(600 \mathrm{MHz} \mathrm{CDCl}_{3}\right): \delta=7.80(\mathrm{dd}, \mathrm{J}=8.2,1.8 \mathrm{~Hz}$, $1 \mathrm{H}), 7.59(\mathrm{~d}, \mathrm{~J}=1.8 \mathrm{~Hz}, 1 \mathrm{H}), 6.90(\mathrm{~d}, \mathrm{~J}=8.2 \mathrm{~Hz}, 1 \mathrm{H})$, $6.81(\mathrm{~d}, \mathrm{~J}=8.4 \mathrm{~Hz}, 1 \mathrm{H}), 6.71(\mathrm{~d}, \mathrm{~J}=2.4 \mathrm{~Hz}, 1 \mathrm{H}), 6.63(\mathrm{dd}$, $\mathrm{J}=8.4,2.4 \mathrm{~Hz}, 1 \mathrm{H}), 6.08(\mathrm{~s}, 2 \mathrm{H}), 6.00(\mathrm{~s}, 2 \mathrm{H}) ;{ }^{13} \mathrm{C} \mathrm{NMR}$ $\left(150 \mathrm{MHz}, \mathrm{CDCl}_{3}\right): \delta=164.82,152.20,147.90,145.36$, $126.19,123.30,114.06,109.92,108.15,108.01,103.92$, 101.97, 101.71; MS (ESI) $m / z 287.5(\mathrm{M}+\mathrm{H})^{+}$, calcd. for $\mathrm{C}_{15} \mathrm{H}_{11} \mathrm{O}_{6} m / z=287.0$.

\section{4-Allyl-2-methoxyphenyl benzo[d][1,3]dioxole-5-carboxylate (1d)}

${ }^{1} \mathrm{H} \quad \mathrm{NMR}\left(600 \mathrm{MHz}, \mathrm{CDCl}_{3}\right): \delta=7.83$ (dd, $J=8.2$, $1.8 \mathrm{~Hz}, 1 \mathrm{H}), 7.62(\mathrm{~d}, J=1.8 \mathrm{~Hz}, 1 \mathrm{H}), 7.26(\mathrm{~s}, 1 \mathrm{H}), 7.04(\mathrm{~d}$, $J=8.0 \mathrm{~Hz}, 1 \mathrm{H}), 6.89(\mathrm{~d}, J=8.2 \mathrm{~Hz}, 1 \mathrm{H}), 6.82(\mathrm{~d}, J=1.8 \mathrm{~Hz}$, $1 \mathrm{H}), 6.06$ (s, 2H), 6.00-5.95 (m, 1H), 5.16-5.06 (m, 2H), $3.80(\mathrm{~s}, 3 \mathrm{H}), 3.40(\mathrm{~d}, J=7.2 \mathrm{~Hz}, 2 \mathrm{H}) ;{ }^{13} \mathrm{C}$ NMR $(150 \mathrm{MHz}$, $\left.\mathrm{CDCl}_{3}\right): \delta=164.24,152.02,151.10,147.80,138.96$, $138.21,137.11,126.27,123.40,122.66,120.71,116.12$, $112.83,110.12,108.09,101.88,55.89,40.12$; MS (ESI) $\mathrm{m} / z$ $335.6(\mathrm{M}+\mathrm{Na})^{+}$, calcd. for $\mathrm{C}_{18} \mathrm{H}_{16} \mathrm{NaO}_{5} \mathrm{~m} / z=335.1$.

\section{2,6-Dimethoxyphenyl benzo[d][1,3]dioxole-5-carboxylate \\ (1e)}

${ }^{1} \mathrm{H} \mathrm{NMR}\left(600 \mathrm{MHz}, \mathrm{CDCl}_{3}\right): \delta=7.86(\mathrm{dd}, J=8.2,1.8 \mathrm{~Hz}$, $1 \mathrm{H}), 7.66(\mathrm{~d}, J=1.8 \mathrm{~Hz}, 1 \mathrm{H}), 7.17-7.16(\mathrm{~m}, 1 \mathrm{H}), 6.89$ $(\mathrm{d}, J=8.2 \mathrm{~Hz}, 1 \mathrm{H}), 6.64(\mathrm{~d}, J=8.4 \mathrm{~Hz}, 1 \mathrm{H}), 6.06(\mathrm{~s}, 2 \mathrm{H})$, $3.80(\mathrm{~s}, 6 \mathrm{H}) ;{ }^{13} \mathrm{C}$ NMR ${ }^{1} \mathrm{H}$ NMR (150 MHz, CDCl $)$ : $\delta=163.88,152.57,151.98,147.76,128.95,126.41,126.23$, $123.32,110.29,108.07,104.95,101.85,56.19$; MS (ESI) $m / z 325.5(\mathrm{M}+\mathrm{Na})^{+}$, calcd. for $\mathrm{C}_{16} \mathrm{H}_{14} \mathrm{NaO}_{6} m / z=325.1$.

\section{2-Acetyl-5-methoxyphenyl benzo[d][1,3] dioxole-5-carboxylate (1f)}

${ }^{1} \mathrm{H} \mathrm{NMR}\left(600 \mathrm{MHz}, \mathrm{CDCl}_{3}\right): \delta=7.89(\mathrm{~d}, J=8.8 \mathrm{~Hz}, 1 \mathrm{H})$, $7.84(\mathrm{dd}, J=8.2,1.8 \mathrm{~Hz}, 1 \mathrm{H}), 7.62(\mathrm{~d}, J=1.8 \mathrm{~Hz}, 1 \mathrm{H}), 6.92$ $(\mathrm{d}, J=8.2 \mathrm{~Hz}, 1 \mathrm{H}), 6.87(\mathrm{dd}, J=8.8,2.4 \mathrm{~Hz}, 1 \mathrm{H}), 6.70(\mathrm{~d}$, $J=2.4 \mathrm{~Hz}, 1 \mathrm{H}), 6.08(\mathrm{~s}, 3 \mathrm{H}), 3.87$ (s, 3H), 2.49 (s, 3H); ${ }^{13} \mathrm{C}$ NMR $\left(150 \mathrm{MHz}, \mathrm{CDCl}_{3}\right): \delta=195.68,164.42,163.73$,
152.39, 151.72, 147.98, 132.37, 126.49, 111.97, 110.07, 109.25, 108.30, 102.01, 55.75, 29.52; MS (ESI) $\mathrm{m} / z 337.4$ $(\mathrm{M}+\mathrm{Na})^{+}$, calcd. for $\mathrm{C}_{17} \mathrm{H}_{14} \mathrm{NaO}_{6} \mathrm{~m} / z=337.1$.

\section{2-(Methoxycarbonyl)phenyl benzo[d][1,3] dioxole-5-carboxylate (1g)}

${ }^{1} \mathrm{H} \mathrm{NMR}\left(600 \mathrm{MHz}, \mathrm{CDCl}_{3}\right): \delta=8.06(\mathrm{dd}, J=7.8,1.8 \mathrm{~Hz}$, $1 \mathrm{H}), 7.85(\mathrm{dd}, J=8.2,1.8 \mathrm{~Hz}, 1 \mathrm{H}), 7.64(\mathrm{~d}, J=1.6 \mathrm{~Hz}, 1 \mathrm{H})$, 7.61-7.59 (m, 1H), 7.36-7.34 (m, 1H), $7.22(\mathrm{dd}, J=8.1$, $0.8 \mathrm{~Hz}, 1 \mathrm{H}), 6.92(\mathrm{~d}, J=8.2 \mathrm{~Hz}, 1 \mathrm{H}), 6.08(\mathrm{~s}, 2 \mathrm{H}), 3.76(\mathrm{~s}$, $3 \mathrm{H}) ;{ }^{13} \mathrm{C} \mathrm{NMR}\left(150 \mathrm{MHz}, \mathrm{CDCl}_{3}\right): \delta=165.04,164.68$, $152.19,150.83,147.89,133.80,131.87,126.36,126.00$, $124.00,123.48,123.40,110.07,108.22,101.94,52.21$; MS (ESI) $m / z 323.4(\mathrm{M}+\mathrm{Na})^{+}$, calcd. for $\mathrm{C}_{16} \mathrm{H}_{12} \mathrm{NaO}_{6}$ $m / z=323.1$.

\section{5-Isopropyl-2-methylphenyl 3-(benzo[d][1,3]dioxol-5-yl) acrylate (2a)}

${ }^{1} \mathrm{H}$ NMR $\left(600 \mathrm{MHz}, \mathrm{CDCl}_{3}\right): \delta=7.79(\mathrm{~d}, J=16.2 \mathrm{~Hz}$, $1 \mathrm{H}), 7.17(\mathrm{~d}, J=7.8 \mathrm{~Hz}, 1 \mathrm{H}), 7.11-7.02(\mathrm{~m}, 3 \mathrm{H}), 6.93$ $(\mathrm{s}, 1 \mathrm{H}), 6.03(\mathrm{~s}, 2 \mathrm{H}), 6.84(\mathrm{~d}, J=8.0 \mathrm{~Hz}, 1 \mathrm{H}), 6.48(\mathrm{~d}$, $J=15.6 \mathrm{~Hz}, 2 \mathrm{H}), 6.03$ (s, 2H), 2.91-2.87 (m, 1H), 1.24 (d, $J=7.2 \mathrm{~Hz}, 6 \mathrm{H}) ;{ }^{13} \mathrm{C}$ NMR $\left(150 \mathrm{MHz}, \mathrm{CDCl}_{3}\right): \delta=165.41$, $149.94,149.33,148.03,146.11,128.66,127.37,124.85$, $124.08,119.84,115.08,106.60,101.65,33.58,23.92$, 15.86; MS (ESI) $m / z 325.6(\mathrm{M}+\mathrm{H})^{+}$, calcd. for $\mathrm{C}_{20} \mathrm{H}_{21} \mathrm{O}_{4}$ $m / z=325.1$.

\section{2-Isopropyl-5-methylphenyl 3-(benzo[d][1,3]dioxol-5-yl) acrylate (2b)}

${ }^{1} \mathrm{H}$ NMR $\left(600 \mathrm{MHz}, \mathrm{CDCl}_{3}\right): \delta=7.78(\mathrm{~d}, J=16.2 \mathrm{~Hz}$, $1 \mathrm{H}), 7.22(\mathrm{~d}, J=7.8 \mathrm{~Hz}, 1 \mathrm{H}), 7.13-7.01(\mathrm{~m}, 4 \mathrm{H}), 6.87(\mathrm{~d}$, d, $J=7.8 \mathrm{~Hz}, 1 \mathrm{H}), 6.48(\mathrm{~d}, J=15.6 \mathrm{~Hz}, 1 \mathrm{H}), 6.03(\mathrm{~s}, 2 \mathrm{H})$, 3.05-3.01 (m, 1H), $2.33(\mathrm{~s}, 3 \mathrm{H}) 1.21(\mathrm{~d}, J=7.2 \mathrm{~Hz}, 6 \mathrm{H})$; ${ }^{13} \mathrm{C} \mathrm{NMR}\left(150 \mathrm{MHz}, \mathrm{CDCl}_{3}\right): \delta=165.88,149.95,148.45$, $147.99,146.12,137.21,136.55,128.65,127.07,124.87$, $122.81,115.16,108.63,106.63,101.65,27.16,23.08$, 20.86; MS (ESI) $m / z 325.5(\mathrm{M}+\mathrm{H})^{+}$, calcd. for $\mathrm{C}_{20} \mathrm{H}_{21} \mathrm{O}_{4}$ $m / z=325.1$.

\section{Benzo[d][1,3]dioxol-5-yl 3-(benzo[d][1,3]dioxol-5-yl)acrylate}

(2c)

${ }^{1} \mathrm{H}$ NMR $\left(600 \mathrm{MHz}, \mathrm{CDCl}_{3}\right): \delta=7.68(\mathrm{~d}, J=15.6 \mathrm{~Hz}$, $1 \mathrm{H}), 7.04-6.97(\mathrm{~m}, 2 \mathrm{H}), 6.77(\mathrm{~d}, J=8.0 \mathrm{~Hz}, 1 \mathrm{H}), 6.73(\mathrm{~d}$, $J=8.4 \mathrm{~Hz}, 1 \mathrm{H}), 6.61(\mathrm{~d}, J=2.4 \mathrm{~Hz}, 1 \mathrm{H}), 6.53(\mathrm{dd}, J=8.4$, $2.4 \mathrm{~Hz}, 1 \mathrm{H}), 5.96(\mathrm{~s}, 2 \mathrm{H}), 5.92(\mathrm{~s}, 2 \mathrm{H}) .5 .84(\mathrm{~s}, 1 \mathrm{H}) ;{ }^{13} \mathrm{C}$ NMR (150 MHz, $\left.\mathrm{CDCl}_{3}\right): \delta=165.90,150.02,148.46$, $148.00,146.33,145.29,145.15,128.60,124.93,114.97$, $114.01,108.65,108.00,106.60,103.86,101.69,101.18$; MS (ESI) $m / z \quad 313.5(\mathrm{M}+\mathrm{H})^{+}$, calcd. for $\mathrm{C}_{17} \mathrm{H}_{13} \mathrm{O}_{6}$ $m / z=313.1$. 
4-Allyl-2-methoxyphenyl 3-(benzo[d][1,3]dioxol-5-yl)acrylate (2d)

${ }^{1} \mathrm{H}$ NMR $\left(600 \mathrm{MHz}, \mathrm{CDCl}_{3}\right): \delta=7.70(\mathrm{~d}, J=15.6 \mathrm{~Hz}, 1 \mathrm{H})$, 7.05-6.92 (m, 3H), 6.79-6.69 (m, 3H), $6.42(\mathrm{~d}, J=15.9 \mathrm{~Hz}$, $1 \mathrm{H})$, 5.98-5.85 (m, 3H), 5.09-5.00 (m, 2H), $3.76(\mathrm{~s}, 3 \mathrm{H})$, $3.33(\mathrm{~d}, J=6.7 \mathrm{~Hz}, 2 \mathrm{H}) ;{ }^{13} \mathrm{C} \mathrm{NMR}\left(150 \mathrm{MHz}, \mathrm{CDCl}_{3}\right)$ : $\delta=165.32,151.04,149.87,148.41,146.15,138.92,138.03$, $137.10,128.76,124.83,122.67,120.71,116.15,114.97$, $112.76,108.61,106.65,101.62,55.90,40.14 ; \mathrm{MS}$ (ESI) $\mathrm{m} / \mathrm{z}$ $361.6(\mathrm{M}+\mathrm{Na})^{+}$, calcd. for $\mathrm{C}_{20} \mathrm{H}_{18} \mathrm{NaO}_{5} \mathrm{~m} / z=361.1$.

\section{2,6-Dimethoxyphenyl 3-(benzo[d][1,3]dioxol-5-yl)acrylate (2e)}

${ }^{1} \mathrm{H}$ NMR $\left(600 \mathrm{MHz}, \mathrm{CDCl}_{3}\right): \delta=7.72(\mathrm{~d}, J=15.6 \mathrm{~Hz}$, $1 \mathrm{H}), 7.10-7.07(\mathrm{~m}, 1 \mathrm{H}), 7.03(\mathrm{~d}, J=1.8 \mathrm{~Hz}, 1 \mathrm{H}), 7.00$ $(\mathrm{d}, J=7.5 \mathrm{~Hz}, 1 \mathrm{H}), 6.76(\mathrm{~d}, J=7.8 \mathrm{~Hz}, 1 \mathrm{H}), 6.57(\mathrm{~d}$, $J=9.0 \mathrm{~Hz}, 2 \mathrm{H}), 6.48(\mathrm{~d}, J=16.2 \mathrm{~Hz}, 1 \mathrm{H}), 5.96(\mathrm{~s}, 2 \mathrm{H}), 3.76$ $(\mathrm{s}, 6 \mathrm{H}) ;{ }^{13} \mathrm{C} \mathrm{NMR}\left(150 \mathrm{MHz}, \mathrm{CDCl}_{3}\right): \delta=164.89,152.51$, 149.82, 148.37, 146.23, 128.85, 126.22, 124.83, 114.83, 108.59, 106.69, 104.92, 101.60, 56.21; MS (ESI) $m / z 351.5$ $(\mathrm{M}+\mathrm{Na})^{+}$, calcd. for $\mathrm{C}_{18} \mathrm{H}_{16} \mathrm{NaO}_{6} \mathrm{~m} / z=351.1$.

\section{2-Acetyl-5-methoxyphenyl 3-(benzo[d][1,3]dioxol-5-yl) acrylate (2f)}

${ }^{1} \mathrm{H}$ NMR $\left(600 \mathrm{MHz}, \mathrm{CDCl}_{3}\right): \delta=7.89(\mathrm{~d}, J=9.0 \mathrm{~Hz}, 1 \mathrm{H})$, $7.82(\mathrm{~d}, J=15.6 \mathrm{~Hz}, 1 \mathrm{H}), 7.13(\mathrm{~d}, J=1.6 \mathrm{~Hz}, 1 \mathrm{H}), 7.11-$ $7.09(\mathrm{~m}, 1 \mathrm{H}), 6.87(\mathrm{dd}, J=8.6,2.4 \mathrm{~Hz}, 2 \mathrm{H}), 6.70(\mathrm{~s}, 1 \mathrm{H})$, $6.52(\mathrm{~d}, J=15.6 \mathrm{~Hz}, 1 \mathrm{H}), 6.06(\mathrm{~s}, 2 \mathrm{H}), 3.88(\mathrm{~s}, 3 \mathrm{H}), 2.54$ $(\mathrm{s}, 3 \mathrm{H}) ;{ }^{13} \mathrm{C}$ NMR $\left(150 \mathrm{MHz}, \mathrm{CDCl}_{3}\right): \delta=195.96,165.36$, $163.69,151.51,150.14,148.45,147.07,132.26,128.51$, $125.18,123.68,114.68,111.89,109.12,108.64,106.71$, 101.69, 55.74, 29.61; MS (ESI) $m / z 363.5(\mathrm{M}+\mathrm{Na})^{+}$, calcd. for $\mathrm{C}_{19} \mathrm{H}_{16} \mathrm{NaO}_{6} m / z=363.1$.

\section{Methyl 2-((3-(benzo[d][1,3]dioxol-5-yl)acryloyl)oxy)benzoate (2g)}

${ }^{1} \mathrm{H}$ NMR $\left(600 \mathrm{MHz}, \mathrm{CDCl}_{3}\right): \delta=7.97(\mathrm{~d}, J=9.0 \mathrm{~Hz}, 1 \mathrm{H})$, $7.73(\mathrm{~d}, J=15.6 \mathrm{~Hz}, 1 \mathrm{H}), 7.53-7.50(\mathrm{~m}, 1 \mathrm{H}), 7.27(\mathrm{~d}$, $J=8.0 \mathrm{~Hz}, 1 \mathrm{H}), 7.19(\mathrm{~s}, 2 \mathrm{H}), 7.11(\mathrm{~d}, J=7.8 \mathrm{~Hz}, 1 \mathrm{H}), 7.04-$ $7.00(\mathrm{~m}, 2 \mathrm{H}), 6.77(\mathrm{~d}, J=8.0 \mathrm{~Hz}, 1 \mathrm{H}), 6.45(\mathrm{~d}, J=15.6 \mathrm{~Hz}$, $1 \mathrm{H}), 5.96(\mathrm{~s}, 2 \mathrm{H}), 3.77(\mathrm{~s}, 3 \mathrm{H}) ;{ }^{13} \mathrm{C} \mathrm{NMR}(150 \mathrm{MHz}$, $\left.\mathrm{CDCl}_{3}\right): \delta=165.61,165.14,150.65,149.99,148.43$, $146.55,133.79,131.77,128.67,125.94,125.01,123.91$, 123.56, 114.87, 108.61, 106.70, 101.66, 52.25; MS (ESI) $m / z$ 349.4 $(\mathrm{M}+\mathrm{Na})^{+}$, calcd. for $\mathrm{C}_{18} \mathrm{H}_{14} \mathrm{NaO}_{6} m / z=349.1$.

\section{5-Isopropyl-2-methylphenyl 5-(benzo[d][1,3]dioxol-5-yl) penta-2,4-dienoate (3a)}

${ }^{1} \mathrm{H}$ NMR $\left(600 \mathrm{MHz}, \mathrm{CDCl}_{3}\right): \delta=7.83-7.79(\mathrm{~m}, J=15.2$, $10.9 \mathrm{~Hz}, 1 \mathrm{H}), 7.09$ (d, $J=7.8 \mathrm{~Hz}, 1 \mathrm{H}), 6.96-6.95(\mathrm{~m}, 3 \mathrm{H})$, $6.88-6.80(\mathrm{~m}, 3 \mathrm{H}), 6.75-6.70(\mathrm{~m}, 2 \mathrm{H}), 6.09(\mathrm{~d}, J=15.2 \mathrm{~Hz}$,
$1 \mathrm{H}), 5.93(\mathrm{~s}, 2 \mathrm{H}), 2.83-2.79(\mathrm{~m}, 1 \mathrm{H}), 2.08(\mathrm{~s}, 3 \mathrm{H}), 1.16(\mathrm{~d}$, $J=14.6 \mathrm{~Hz}, 6 \mathrm{H}) ;{ }^{13} \mathrm{C} \mathrm{NMR}\left(150 \mathrm{MHz}, \mathrm{CDCl}_{3}\right): \delta=165.51$, $149.31,148.79,148.35,148.04,146.69,141.25,130.88$, $130.40,127.42,124.33,124.11,123.34,119.88,119.20$, 108.63, 105.93, 101.50, 33.61, 24.08, 15.92; MS (ESI) $\mathrm{m} / z$ $373.5(\mathrm{M}+\mathrm{Na})^{+}$, calcd. for $\mathrm{C}_{22} \mathrm{H}_{22} \mathrm{NaO}_{4} m / z=373.2$.

\section{2-Isopropyl-5-methylphenyl 5-(benzo[d][1,3]dioxol-5-yl) penta-2,4-dienoate (3b)}

${ }^{1} \mathrm{H}$ NMR $\left(600 \mathrm{MHz}, \mathrm{CDCl}_{3}\right): \delta=7.55-7.51(\mathrm{~m}, 1 \mathrm{H}), 7.15$ $(\mathrm{d}, J=7.8 \mathrm{~Hz}, 1 \mathrm{H}), 6.96-6.95(\mathrm{~m}, 2 \mathrm{H}), 6.87-6.86(\mathrm{~m}$, $2 \mathrm{H}), 6.79(\mathrm{~s}, 1 \mathrm{H}), 6.74-6.71(\mathrm{~m}, 2 \mathrm{H}), 6.09(\mathrm{~d}, J=15.0 \mathrm{~Hz}$, $1 \mathrm{H}), 5.92(\mathrm{~s}, 2 \mathrm{H}), 2.96-2.91(\mathrm{~m}, 1 \mathrm{H}), 2.24(\mathrm{~s}, 3 \mathrm{H}), 1.12(\mathrm{~d}$, $J=6.6 \mathrm{~Hz}, 6 \mathrm{H}) ;{ }^{13} \mathrm{C} \mathrm{NMR}\left(150 \mathrm{MHz}, \mathrm{CDCl}_{3}\right): \delta=166.02$, $148.81,148.36,147.97,146.71,141.32,137.22,136.60$, $130.40,127.11,126.46,124.33,123.36,122.87,119.27$, $108.64,105.93,101.52,27.17,22.75,20.94$; MS (ESI) $m / z$ $373.5(\mathrm{M}+\mathrm{Na})^{+}$, calcd. for $\mathrm{C}_{22} \mathrm{H}_{22} \mathrm{NaO}_{4} m / z=373.2$.

\section{Benzo[d][1,3]dioxol-5-yl 5-(benzo[d][1,3]dioxol-5-yl) penta-2,4-dienoate (3c)}

${ }^{1} \mathrm{H}$ NMR $\left(600 \mathrm{MHz}, \mathrm{CDCl}_{3}\right): \delta=7.57$ (dd, $J=15.2$, $11.0 \mathrm{~Hz}, 1 \mathrm{H}), 7.02(\mathrm{~d}, J=1.6 \mathrm{~Hz}, 1 \mathrm{H}), 6.94(\mathrm{dd}, J=8.0$, $1.6 \mathrm{~Hz}, 1 \mathrm{H}), 6.88(\mathrm{~d}, J=15.0 \mathrm{~Hz}, 1 \mathrm{H}), 6.81-6.77(\mathrm{~m}, 3 \mathrm{H})$, $6.66(\mathrm{~d}, J=2.4 \mathrm{~Hz}, 1 \mathrm{H}), 6.58(\mathrm{dd}, J=8.4,2.4 \mathrm{~Hz}, 1 \mathrm{H}), 6.09$ $(\mathrm{d}, J=15.2 \mathrm{~Hz}, 1 \mathrm{H}), 5.99(\mathrm{~d}, J=9.6 \mathrm{~Hz}, 4 \mathrm{H}) ;{ }^{13} \mathrm{C} \mathrm{NMR}$ $\left(150 \mathrm{MHz}, \mathrm{CDCl}_{3}\right): \delta=165.84,148.81,148.34,147.95$, $146.76,145.23,145.15,141.34,130.37,124.27,123.27$, $119.07,113.99,108.59,107.96,105.95,103.86,101.65$, 101.45; MS (ESI) $m / z 339.2(\mathrm{M}+\mathrm{H})^{+}$, calcd. for $\mathrm{C}_{19} \mathrm{H}_{15} \mathrm{O}_{6}$ $m / z=339.1$.

\section{4-Allyl-2-methoxyphenyl 5-(benzo[d][1,3]dioxol-5-yl) penta-2,4-dienoate (3d)}

${ }^{1} \mathrm{H}$ NMR $\left(600 \mathrm{MHz}, \mathrm{CDCl}_{3}\right): \delta=7.64-7.60(\mathrm{~m}, 1 \mathrm{H})$, $7.16-7.13(\mathrm{~m}, 1 \mathrm{H}), 7.03(\mathrm{~d}, J=1.8 \mathrm{~Hz}, 1 \mathrm{H}), 6.94(\mathrm{dd}$, $J=8.0,1.8 \mathrm{~Hz}, 1 \mathrm{H}), 6.87(\mathrm{~d}, J=15.6 \mathrm{~Hz}, 1 \mathrm{H}), 6.81-6.78$ $(\mathrm{m}, 2 \mathrm{H}), 6.63(\mathrm{~d}, J=8.4 \mathrm{~Hz}, 2 \mathrm{H}), 6.22(\mathrm{~d}, J=15.2 \mathrm{~Hz}, 1 \mathrm{H})$, $6.00(\mathrm{~s}, 2 \mathrm{H}), 3.82(\mathrm{~s}, 6 \mathrm{H}) ;{ }^{13} \mathrm{C}$ NMR $\left(150 \mathrm{MHz} \mathrm{CDCl}_{3}\right)$ : $\delta=164.83,152.51,148.68,148.31,146.65,140.92,130.53$, $128.80,126.14,124.54,123.14,119.03,108.55,105.96$, 104.92, 101.42, 56.19; MS (ESI) $m / z 365.4(\mathrm{M}+\mathrm{H})^{+}$, calcd. for $\mathrm{C}_{22} \mathrm{H}_{21} \mathrm{O}_{5} m / z=365.1$.

\section{2,6-Dimethoxyphenyl 5-(benzo[d][1,3]dioxol-5-yl) penta-2,4-dienoate (3e)}

${ }^{1} \mathrm{H}$ NMR $\left(600 \mathrm{MHz}, \mathrm{CDCl}_{3}\right): \delta=7.64-7.60(\mathrm{~m}, 1 \mathrm{H})$, $7.16-7.13(\mathrm{~m}, 1 \mathrm{H}), 7.03(\mathrm{~d}, J=1.8 \mathrm{~Hz}, 1 \mathrm{H}), 6.94(\mathrm{dd}$, $J=8.0,1.8 \mathrm{~Hz}, 1 \mathrm{H}), 6.87(\mathrm{~d}, J=15.6 \mathrm{~Hz}, 1 \mathrm{H}), 6.81-6.78$ $(\mathrm{m}, 2 \mathrm{H}), 6.63(\mathrm{~d}, J=8.4 \mathrm{~Hz}, 2 \mathrm{H}), 6.22(\mathrm{~d}, J=15.2 \mathrm{~Hz}, 1 \mathrm{H})$, $6.00(\mathrm{~s}, 2 \mathrm{H}), 3.82(\mathrm{~s}, 6 \mathrm{H}) ;{ }^{13} \mathrm{C} \mathrm{NMR}\left(150 \mathrm{MHz} \mathrm{CDCl}_{3}\right)$ : 
$\delta=164.83,152.51,148.68,148.31,146.65,140.92,130.53$, $128.80,126.14,124.54,123.14,119.03,108.55,105.96$, 104.92, 101.42, 56.19; MS (ESI) $m / z$ 377.4 $(\mathrm{M}+\mathrm{Na})^{+}$, calcd. for $\mathrm{C}_{20} \mathrm{H}_{18} \mathrm{NaO}_{6} m / z=377.1$.

\section{2-Acetyl-5-methoxyphenyl 5-(benzo[d][1,3]dioxol-5-yl) penta-2,4-dienoate (3f)}

${ }^{1} \mathrm{H} \mathrm{NMR}\left(600 \mathrm{MHz}, \mathrm{CDCl}_{3}\right): \delta=7.86(\mathrm{~d}, J=8.8 \mathrm{~Hz}, 1 \mathrm{H})$, $7.63(\mathrm{dd}, J=15.2,11.0 \mathrm{~Hz}, 1 \mathrm{H}), 7.03(\mathrm{~d}, J=1.6 \mathrm{~Hz}, 1 \mathrm{H})$, 6.98-6.87 (m, 3H), 6.86-6.78 (m, 4H), $6.66(\mathrm{~d}, J=2.5 \mathrm{~Hz}$, $1 \mathrm{H}), 6.18$ (d, J=15.2 Hz, 1H), $6.00(\mathrm{~s}, 2 \mathrm{H}), 3.85(\mathrm{~s}, 3 \mathrm{H})$, $2.51(\mathrm{~s}, 3 \mathrm{H}) ;{ }^{13} \mathrm{C} \mathrm{NMR}\left(150 \mathrm{MHz}, \mathrm{CDCl}_{3}\right): \delta=192.87$, $165.23,163.65,151.54,148.89,148.36,147.49,141.78$, $132.19,130.34,124.26,123.71,117.79,111.86,109.06$, 108.60, 105.98, 101.47, 55.71, 29.69; MS (ESI) $m / z 389.4$ $(\mathrm{M}+\mathrm{Na})^{+}$, calcd. for $\mathrm{C}_{21} \mathrm{H}_{18} \mathrm{NaO}_{6} \mathrm{~m} / z=389.1$.

\section{Methyl 2-((5-(benzo[d][1,3]dioxol-5-yl)penta-2,4-dienoyl) oxy)benzoate (3g)}

${ }^{1} \mathrm{H}$ NMR $\left(600 \mathrm{MHz}, \mathrm{CDCl}_{3}\right): \delta=7.95(\mathrm{dd}, J=7.8,1.8 \mathrm{~Hz}$, $1 \mathrm{H})$, 7.59-7.46 (m, 2H), 7.27-7.21 (m, 1H), $7.08(\mathrm{dd}$, $J=8.2,0.8 \mathrm{~Hz}, 1 \mathrm{H}), 6.95(\mathrm{~d}, J=1.6 \mathrm{~Hz}, 1 \mathrm{H}), 6.87$ (dd, $J=8.0,1.6 \mathrm{~Hz}, 1 \mathrm{H}), 6.81(\mathrm{~d}, J=15.6 \mathrm{~Hz}, 1 \mathrm{H}), 6.76-6.68$ (m, 2H), 6.12 (d, J=15.2 Hz, 1H), $5.92(\mathrm{~s}, 4 \mathrm{H}), 3.76$ (s, $2 \mathrm{H}) ;{ }^{13} \mathrm{C}$ NMR $\left(150 \mathrm{MHz}, \mathrm{CDCl}_{3}\right): \delta=164.51,164.12$, $149.61,147.76,147.31,145.94,140.29,132.71,130.70$, $129.39,124.85,123.40,122.89,122.53,122.22,118.00$,
107.55, 104.93, 100.43, 51.20; MS (ESI) $\mathrm{m} / z \quad 375.5$ $(\mathrm{M}+\mathrm{Na})^{+}$, calcd. for $\mathrm{C}_{20} \mathrm{H}_{16} \mathrm{NaO}_{6} \mathrm{~m} / z=375.1$.

\section{Biological assay}

The in vitro fungicidal activities of the target compounds 1a-3g against Rhizoctonia solani, Fusarium graminearum, Phomopsis adianticola, Alternaria tenuis Nees, Phytophthora capsici and Gloeosporium theae-sinensis were evaluated using mycelium growth rate test, and all the procedure for bioassay were according to the methods reported in literature [23].

\section{Results and discussion}

\section{Synthesis}

A series of novel compounds $1 \mathbf{a}-\mathbf{g}, \mathbf{2 a}-\mathbf{g}$ and $3 \mathbf{a}-\mathbf{g}$ derived from natural phenolic compounds existed in essential oils based on piperine scaffold can be synthesized by a mild and simple method as described in Scheme 1. In brief, the intermediate $(E)$-3-(benzo $[d][1,3]$ dioxol-5-yl)acrylic acid $(n=1)$ can be prepared using piperonal as starting materials [21], and the other intermediate piperic acid $(n=2)$ was synthesized via basic hydrolysis reaction of piperine [22]. Then, all three acids were coupling with various essential oils molecules to obtain the corresponding esters using an optimization method.

$$
\text { n=0,1,2 }
$$

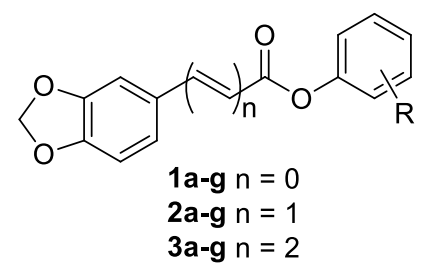<smiles>O=C(O)/C=C/c1ccc2c(c1)OOO2</smiles>

(E)-3-(Benzo[d][1,3]dioxol-5yl)acrylic acid $(n=1)$

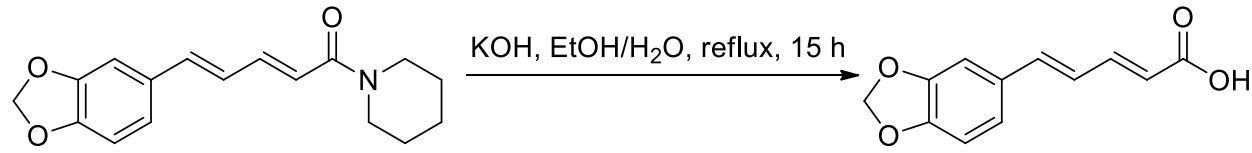

Piperine

Piperic acid $(n=2)$

Scheme 1 Synthetic route for intermediates and target molecules 
Table 1 The optimal reaction conditions of piperic acid and carvacrol

\begin{tabular}{llllll}
\hline $\begin{array}{l}\text { Entry } \\
\text { Catalytic } \\
\text { system }\end{array}$ & Solvent & $\begin{array}{l}\text { Temperature } \\
\left({ }^{\circ} \mathbf{C}\right)\end{array}$ & Time (h) & Yield (\%) \\
\hline 1 & DCC/DMAP & THF & 40 & 6 & 47.47 \\
2 & DCC/DMAP & DCM & 40 & 6 & 78.42 \\
3 & DCC/DMAP & MeCN & 40 & 6 & 83.40 \\
4 & DCC/DMAP & MeCN & 60 & 6 & 68.55 \\
5 & DCC/DMAP & MeCN & 90 & 6 & 65.46 \\
6 & EDCI/HOBT & MeCN & 40 & 6 & NR \\
7 & CDI/DIPEA & MeCN & 40 & 6 & NR \\
\hline
\end{tabular}

To achieve the above goal for these essential oil derivatives, the initial experiment was optimized, and the different reaction conditions have been explored (Table 1). As can be seen from Table 1 (Entry 6 and 7), when the condensation system is EDCI/HOBT or CDI/DIPEA, TLC analysis showed that no obvious product was produced, however, the yields are improved when the condensation reactions are performed under the DCC/ DMAP system. With this condition (DCC/DMAP) in hand, the solvent is further screened, and an equal volume of acetonitrile, tetrahydrofuran and dichloromethane are used as solvents. The reaction time and temperature are the same. The relationship between solvent and yield was obtained, as shown in Table 1, when acetonitrile was the solvent, the yield was the highest. In order to investigate the effect of the target compound yield on the reaction temperature, the experiment was carried out at a reaction temperature of $40{ }^{\circ} \mathrm{C}, 60{ }^{\circ} \mathrm{C}$, and $90{ }^{\circ} \mathrm{C}$, respectively. The results show that the yield gradually decreases with increasing temperature, and the yield is highest at $40{ }^{\circ} \mathrm{C}$. Finally, we determined the optimal synthetic conditions for the synthesis of pepper acid-directed essential oil derivatives: DCC/DMAP is a catalytic condensation system, the solvent is acetonitrile, the reaction temperature is $40{ }^{\circ} \mathrm{C}$, and the yield of the target compound is $83.40 \%$.

All of the new natural phenolic derivatives were synthesized according to the optimal conditions described above, and the structures of all the obtained compounds in this study were confirmed by satisfactory spectral analysis, including ${ }^{1} \mathrm{H}$ NMR, ${ }^{13} \mathrm{C}$ NMR, ESI-MS. The chemical formulas of all compounds were described in
Table 2, and their chemical structures and basic physicochemical properties were summarized in "Materials and methods".

\section{Spectrum analyses}

The structures of all target compounds $1 \mathbf{a}-\mathbf{3 g}$ were confirmed by ${ }^{1} \mathrm{H}$ NMR, ${ }^{13} \mathrm{C}$ NMR (Additional file 1) and mass spectrometry, and their structures were well consistent with all the spectral data. A representative ${ }^{1} \mathrm{H}$ NMR spectrum of $\mathbf{1 c}$ is shown in Fig. 2, and each hydrogen shows a characteristic absorption peak. The methylene group on the piperine skeleton was not affected by other $\mathrm{H}$ in the ortho position, and a single peak appeared at $6.06 \mathrm{ppm}$, and the $\mathrm{H}$ of the benzene ring showed between 7.81 and $6.62 \mathrm{ppm}$.

\section{Biological activity \\ Primary screening test}

In this study, all essential oil derivatives $1 \mathbf{a}-\mathbf{g}, \mathbf{2 a}-\mathbf{g}$, and $3 \mathbf{a}-\mathbf{g}$ were screened for their antifungal activities in vitro against six common plant pathogenic fungi (Rhizoctonia solani, Fusarium graminearum, Alternaria tenuis Nees, Gloeosporium theae-sinensis, Phytophthora capsici, Phomopsis adianticola), and the preliminary screening results were outlined in Table 3 .

Generally, as shown in Table 3, the preliminary assay illustrated that some compounds of the essential oil derivatives based on piperine displayed good inhibitory activities against some tested fungal strains, and we also can find that some of the target compounds have better inhibitory activities than piperine and carbendazim at the concentration of $100 \mu \mathrm{g} / \mathrm{mL}$. Notably, six compounds displayed fungicidal activity more than $40 \%$ against Rhizoctonia solani, especially compound 1f displayed an $65.00 \%$ inhibition rate, better than that of piperine (63.13\%). Three compounds displayed fungicidal activity more than $40 \%$ against Fusarium graminearum, except compound $\mathbf{2 b}$ displayed an $62.61 \%$ inhibition rate, better than that of piperine (53.04\%). Four compounds displayed fungicidal activity more than $40 \%$ against Alternaria tenuis Nees, except compound 1d displayed $71.07 \%$ inhibition rate, better than that of the piperine $(66.12 \%)$ and carbendazim (13.22\%). Five compounds displayed fungicidal activity more than $40 \%$ against Gloeosporium theae-sinensis, 2b displayed an $66.92 \%$ inhibition rate, which is less than the activity of piperine (76.92\%). Four compounds 
Table 2 The chemical structure of target compounds $1 \mathrm{a}-\mathbf{3 g}$

\begin{tabular}{|c|c|c|c|c|}
\hline Compd. no. & Structure & Appearance & MP $\left({ }^{\circ} \mathrm{C}\right)$ & Yield (\%) \\
\hline $1 \mathrm{a}$ & & $\begin{array}{l}\text { Yellowish } \\
\text { liquid }\end{array}$ & - & 83.4 \\
\hline $1 b$ & & White solid & $61-63$ & 66.0 \\
\hline $1 \mathrm{c}$ & & White solid & $117-120$ & 42.3 \\
\hline $1 d$ & & Yellowish solid & $68-72$ & 78.85 \\
\hline $1 \mathrm{e}$ & & White solid & $158-160$ & 57.7 \\
\hline 1f & & White solid & $116-120$ & 88.46 \\
\hline $1 \mathrm{~g}$ & & White solid & $93-96$ & 83.90 \\
\hline $2 \mathrm{a}$ & & $\begin{array}{l}\text { Brownish } \\
\text { yellow } \\
\text { semisolid }\end{array}$ & $45-49$ & 81.20 \\
\hline $2 b$ & & White solid & $70-73$ & 75.6 \\
\hline $2 \mathrm{c}$ & & White solid & $134-137$ & 80.3 \\
\hline $2 d$ & & White solid & $121-125$ & 77.8 \\
\hline $2 \mathrm{e}$ & & White solid & $174-178$ & 85.6 \\
\hline $2 \mathrm{f}$ & & White solid & $137-141$ & 77.8 \\
\hline
\end{tabular}


Table 2 (continued)

\begin{tabular}{|c|c|c|c|c|}
\hline Compd. no. & Structure & Appearance & $\operatorname{MP}\left({ }^{\circ} \mathrm{C}\right)$ & Yield (\%) \\
\hline $2 \mathrm{~g}$ & & White solid & $122-125$ & 84.6 \\
\hline $3 a$ & & $\begin{array}{c}\text { Yellow } \\
\text { semisolid }\end{array}$ & $68-72$ & 55.8 \\
\hline $3 b$ & & Yellowish solid & $89-91$ & 73.5 \\
\hline $3 \mathrm{c}$ & & Yellowish solid & $136-138$ & 59.3 \\
\hline $3 d$ & & Yellowish solid & $109-113$ & 62.13 \\
\hline $3 \mathrm{e}$ & & $\begin{array}{l}\text { Orange-yellow } \\
\text { solid }\end{array}$ & $155-158$ & 53.8 \\
\hline $3 f$ & & $\begin{array}{l}\text { Orange-yellow } \\
\text { solid }\end{array}$ & $127-130$ & 53.0 \\
\hline $3 g$ & & Yellow solid & $115-118$ & 40.06 \\
\hline
\end{tabular}

displayed fungicidal activity more than $40 \%$ against Phytophthora capsici, except compound $\mathbf{2 b}$ displayed an $100 \%$ inhibition rate, which is much greater than the piperine $(41.88 \%)$ and carbendazim (34.27\%). Four compounds displayed fungicidal activity more than 40\% against Phomopsis adianticola, except compound 2b displayed an 100\% inhibition rate, far superior to the piperine (29.63\%).

\section{Secondary screening test}

The preliminary assay indicated many of the target compounds exhibited good fungicidal activities compared to the commercial fungicide carbendazim, in order to further investigate the potential fungicidal activities, we thus selected some compounds like 1a, 1b, 1c, 1d, 1g, 2a, 2b, 2g to have further exploration in such a situation, and compared the values of $\mathrm{IC}_{50}$ with piperine and carbendazim at different concentrations. The fungicidal activities expressed as $\mathrm{IC}_{50}$ values for highly potential compounds are listed in Table 4, which indicated some compounds had good inhibitory effects. As shown in Table 4, compounds 1a, 1g, 2b, $2 \mathbf{g}\left(\mathrm{IC}_{50}=11.21,87.66,7.79,97.84 \mu \mathrm{g} / \mathrm{mL}\right)$ all displayed good inhibitory effects on Phytophthora capsici compared with the positive control carbendazim $\left(\mathrm{IC}_{50}>100 \mu \mathrm{g} / \mathrm{mL}\right)$. Compounds $\mathbf{1 a}$ and $\mathbf{2 b}$ displayed good inhibitory effects compared with the piperine $\left(\mathrm{IC}_{50}=34.87 \mu \mathrm{g} / \mathrm{mL}\right)$. In particular, $2 \mathbf{b}$ exhibits a broad spectrum of bacteriostatic activity. 


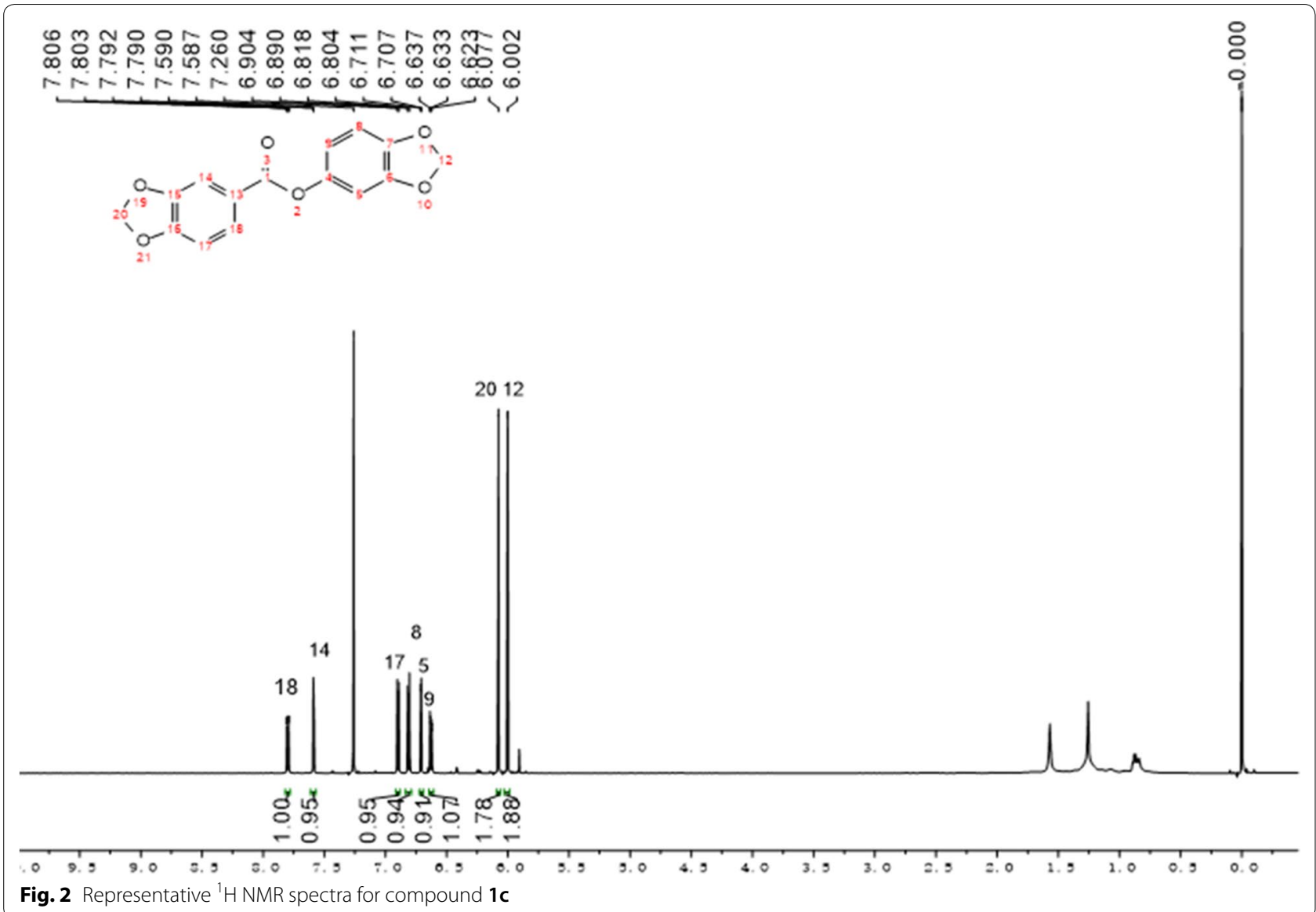

In addition, the Fig. 3 indicated the inhibition effects of target compounds 1a, $\mathbf{2 b}$ on Phomopsis adianticola compared with that of piperine and carbendazim, which confirmed that the compounds $\mathbf{1 a}$ and $\mathbf{2 b}$ displayed the superior fungicidal activities on the Phomopsis adianticola at different concentrations of 12.5, 25, 50, 100, $200 \mu \mathrm{g} / \mathrm{mL}$.

\section{Conclusions}

In summary, 21 piperine-directed essential oil derivatives have been designed, synthesized and evaluated as potential fungicides. The structures of all obtained molecules were characterized by ${ }^{1} \mathrm{H}-\mathrm{NMR},{ }^{13} \mathrm{C}-\mathrm{NMR}$ and ESIMS spectra analyses, and potential bioactivity was also assessed. Preliminary bioassay results indicate that some new compounds show better fungistatic activity than piperine. Among them, compound $\mathbf{2 b}$ exhibits a broad spectrum of fungicidal activity, and it is hoped that further development of a new piperine-oriented agrochemicals. 
Table 3 In vitro fungicidal activity of target compounds $1 \mathrm{a}-\mathbf{3 g}$

\begin{tabular}{|c|c|c|c|c|c|c|c|}
\hline \multirow[t]{2}{*}{ Entry } & \multirow[t]{2}{*}{ Compd. no. } & \multicolumn{6}{|c|}{ In vitro fungicidal activity (\%)/100 $\mu \mathrm{g} / \mathrm{mL}$} \\
\hline & & R.S $S^{\mathrm{a}}$ & $F . G^{\mathrm{a}}$ & $A . T^{\mathrm{a}}$ & $G . T^{\mathrm{a}}$ & $P . C^{\mathrm{a}}$ & $P . A^{\mathrm{a}}$ \\
\hline 1 & $1 \mathrm{a}$ & 21.88 & 18.26 & 47.11 & 51.54 & 68.38 & 68.89 \\
\hline 2 & $1 b$ & 29.69 & 36.52 & 52.89 & 35.77 & 31.62 & 61.85 \\
\hline 3 & $1 c$ & 57.50 & 0.00 & -1.65 & 4.62 & 14.53 & 3.70 \\
\hline 4 & $1 d$ & 30.63 & 17.39 & 71.07 & 52.31 & 24.79 & 40.00 \\
\hline 5 & $1 e$ & 17.50 & 15.65 & 25.62 & 27.69 & 29.06 & 3.70 \\
\hline 6 & $1 \mathrm{f}$ & 65.00 & 31.74 & 33.06 & 26.92 & 30.77 & 47.41 \\
\hline 7 & $1 \mathrm{~g}$ & 20.94 & 22.61 & 7.44 & 0.00 & 47.86 & 23.70 \\
\hline 8 & $2 a$ & 18.13 & 34.78 & 34.71 & 43.08 & 35.90 & 45.19 \\
\hline 9 & $2 b$ & 57.50 & 62.61 & 52.07 & 66.92 & 100 & 100.00 \\
\hline 10 & $2 c$ & 35.94 & 18.26 & 7.44 & 15.38 & 35.90 & -3.70 \\
\hline 11 & $2 d$ & 37.19 & 19.13 & 7.44 & 18.08 & 38.46 & 0.00 \\
\hline 12 & $2 e$ & 63.75 & 21.74 & -3.31 & 3.08 & 27.35 & 3.70 \\
\hline 13 & $2 f$ & 40.94 & 20.87 & 2.48 & 13.85 & 33.33 & -2.22 \\
\hline 14 & $2 g$ & 46.56 & 11.30 & 9.92 & 60.77 & 47.01 & 29.63 \\
\hline 15 & $3 a$ & 30.94 & 46.09 & 23.97 & 30.77 & 33.33 & 18.52 \\
\hline 16 & $3 b$ & 25.00 & 35.65 & 25.62 & 15.38 & 23.08 & 16.30 \\
\hline 17 & $3 c$ & 20.94 & 35.65 & 13.22 & 15.00 & 5.98 & 3.70 \\
\hline 18 & $3 d$ & 21.25 & 45.22 & 24.79 & 38.46 & 19.66 & 17.04 \\
\hline 19 & $3 e$ & 15.63 & 33.04 & 16.53 & 24.62 & 24.79 & 18.52 \\
\hline 20 & $3 f$ & 7.81 & 37.09 & 16.53 & 23.08 & 0.85 & 29.63 \\
\hline 21 & $3 g$ & 18.13 & 24.35 & 17.36 & 30.77 & 15.38 & 29.63 \\
\hline 22 & Piperine & 63.13 & 53.04 & 66.12 & 76.92 & 41.88 & 29.63 \\
\hline 23 & Carbendazim & 100.00 & 100.00 & 13.22 & 100.00 & 34.27 & 100.00 \\
\hline
\end{tabular}

Table 4 The $\mathrm{IC}_{50}$ of some compounds against the plant pathogen fungi

\begin{tabular}{|c|c|c|c|c|c|c|c|}
\hline \multirow[t]{2}{*}{ Entry } & \multirow[t]{2}{*}{ Compd. no. } & \multicolumn{6}{|c|}{$I C_{50}^{a}(\mu \mathrm{g} / \mathrm{mL})$} \\
\hline & & $R \cdot S^{b}$ & F.G & A.T & G.T & $P . C$ & $P . A$ \\
\hline 1 & $1 a$ & 39.92 & 156.99 & 43.06 & 64.65 & 11.21 & 35.67 \\
\hline 2 & $1 b$ & 29.29 & $>200$ & 121.77 & $>200$ & - & 45.75 \\
\hline 3 & $1 c$ & 69.06 & - & - & - & - & - \\
\hline 4 & $1 d$ & - & - & - & 142.36 & - & - \\
\hline 5 & $1 \mathrm{~g}$ & - & - & - & - & 87.66 & - \\
\hline 6 & $2 a$ & 89.50 & - & - & - & - & 72.66 \\
\hline 7 & $2 b$ & 39.46 & 38.83 & 12.02 & 22.55 & 7.79 & 8.84 \\
\hline 8 & $2 g$ & - & - & $>200$ & 81.95 & 97.54 & - \\
\hline 9 & Piperine & 89.50 & $>200$ & 116.77 & 42.84 & 34.87 & 84.88 \\
\hline 10 & Carbendazim & 2.94 & 3.30 & 173.18 & 2.86 & 114.42 & 3.73 \\
\hline
\end{tabular}

${ }^{a} \mathrm{IC}_{50}$-compound concentration required to inhibit colony growth by $50 \%$

b R.S, Rhizoctonia solani; F.G, Fusarium graminearum; A.T, Alternaria tenuis Nees; G.T, Gloeosporium theae-sinensis; P.C, Phytophthora capsici; P.A, Phomopsis adianticola

c Carbendazim, used as positive control 


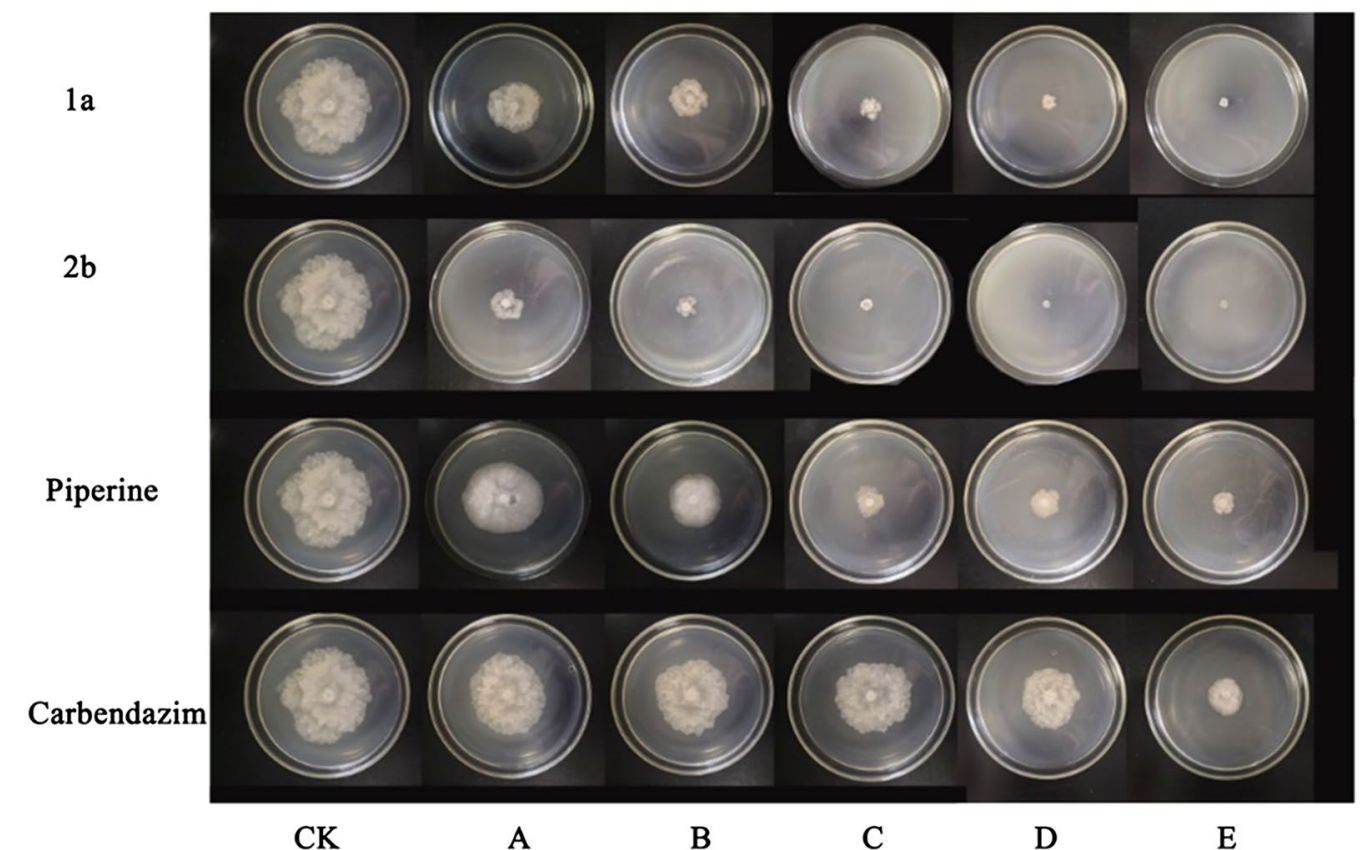

Fig. $\mathbf{3}$ Inhibition activity of compounds $\mathbf{1 a}, \mathbf{2} \mathbf{b}$, piperine and carbendazim on Phomopsis adianticola. $\mathbf{a}-\mathbf{e}$ The concentration of compounds 1a, 2b, piperine and carbendazim are 12.5, 25, 50, 100, and $200 \mu \mathrm{g} / \mathrm{mL}$; CK blank control

\section{Supplementary information}

Supplementary information accompanies this paper at https://doi. org/10.1186/s13065-020-00676-4.

Additional file 1. ${ }^{1} \mathrm{H} N \mathrm{NM}$, and ${ }^{13} \mathrm{C}$ NMR spectra for the target compounds.

\section{Abbreviations}

EOs: Essential oils; m.p.: Melting points; TLC: Analytical thin-layer chromatography; R.S: Rhizoctonia solani; F.G: Fusarium graminearum; A.T: Alternaria tenuis Nees; G.T: Gloeosporium theae-sinensis; P.C: Phytophthora capsici; P.A: Phomopsis adianticola.

\section{Acknowledgements}

The authors are thankful to the Analysis and Testing Center of Huazhong Agricultural University for their support for this research work.

\section{Authors' contributions}

JW (performed all studies and bioassay, and wrote the manuscript), WW (synthesized the selected derivatives), HX (synthesized the selected derivatives), DS (bioactivity evaluation), XC (proposed the project and explained the analyses, and revised the manuscript). All authors read and approved the final manuscript.

\section{Funding}

Financial support from the National Innovation and Entrepreneurship Training Program for College Students (201910504109) is gratefully acknowledged, and the authors also appreciated for Huazhong Agricultural University for provision of excellent lab facilities for this research work.

\section{Availability of data and materials}

All data generated or analyzed during this study are included in this published article.

\section{Competing interests}

The authors declare no conflicts of interest.

Received: 15 November 2019 Accepted: 16 March 2020

Published online: 30 March 2020

\section{References}

1. Mujumdar AM, Dhuley JN, Deshmukh VK, Raman PH, Thorat SL, Naik SR (1990) Effect of piperine on pentobarbitone induced hypnosis in rats. Indian J Exp Biol 28:486-487

2. Kong LD, Cheng CHK, Tan RX (2004) Inhibition of MAO A and B by some plant-derived alkaloids, phenols and anthraquinones. J Ethnopharmacol 91:351-355

3. Pradeep CR, Kuttan G (2002) Effect of piperine on the inhibition of lung metastasis induced B16F-10 melanoma cells in mice. Clin Exp Meta 19:703-708

4. Sunila ES, Kuttan G (2004) Immunomodulatory and antitumor activity of Piper longum Linn. and piperine. J Ethnopharmacol 90:339-346

5. Selvendiran K, Padmavathi R, Magesh V, Sakthisekaran D (2005) Preliminary study on inhibition of genotoxicity by piperine in mice. Fitoterapia 76:296-300

6. Yasir A, Ishtiaq S, Jahangir M, Ajaib M, Salar U, Khan KM (2018) Biologyoriented synthesis (BIOS) of piperine derivatives and their comparative analgesic and antiinflammatory activities. Med Chem 14:269-280

7. Lee SA, Hong SS, Han XH, Hwang JS, Oh GJ, Lee KS, Lee MK, Hwang BY, Ro JS (2005) Piperine from the fruits of Piper longum with inhibitory effect on monoamine oxidase and antidepressant-like activity. Chem Pharm Bull 53:832-835

8. Koul IB, Kapil A (1993) Evaluation of the liver protective potential of piperine, an active principle of black and long peppers. Planta Med 59:413-417

9. Choi BM, Kim SM, Park TK, Li G, Hong SJ, Park R, Chung HT, Kim BR (2007) Piperine protects cisplatin-induced apoptosis via heme oxygenase-1 induction in auditory cells. J Nutr Biochem 18:615-622 
10. Rong A, Bao N, Sun Z, Borjihan G, Qiao Y, Jin Z (2015) Synthesis and antihyperlipidemic activity of piperic acid serivatives. Nat Prod Commun 10:289-290

11. Umadevi P, Deepti K, Venugopal DVR (2013) Synthesis, anticancer and antibacterial activities of piperine analogs. Med Chem Res 22:5466-5471

12. Raut JS, Karuppayil SM, Raut JS, Karuppayil SM (2014) A status review on the medicinal properties of essential oils. Ind Crops Prod 62:250-264

13. Isman M (2006) Botanical insecticides, deterrents, and repellents in modern agriculture and an increasingly regulated world. Ann Rev Entomol 51:45-66

14. Bakkali F, Averbeck S, Averbeck D, Idaomar M (2008) Biological effects of essential oils-a review. Food Chem Toxicol 46:446-475

15. Regnault-Roger C, Vincent C, Arnason JT (2012) Essential oils in insect control: low-risk products in a high-stakes world. Ann Rev Entomol 57:405

16. Machial CM, Ikkei S, Michael S, Roderick B, Isman MB (2010) Evaluation of the toxicity of 17 essential oils against Choristoneura rosaceana (Lepidoptera: Tortricidae) and Trichoplusia ni (Lepidoptera: Noctuidae). Pest Manag Sci 66:1116-1121

17. Mann RS, Tiwari S, Smoot JM, Rouseff RL, Stelinski LL (2012) Repellency and toxicity of plant-based essential oils and their constituents against Diaphorina citri Kuwayama (Hemiptera: Psyllidae). J Appl Entomol 136:87-96
18. Song ZJ, Na ZN, Zhi LQ, Long LZ, Shan DS, Ligang Z, Wei DZ (2011) Repellent constituents of essential oil of Cymbopogon distans aerial parts against two stored-product insects. J Agric Food Chem 59:9910-9915

19. Su H, Wang W, Bao L, Wang S, Cao X (2017) Synthesis and evaluation of essential oil-derived $\beta$-methoxyacrylate derivatives as high potential fungicides. Molecules 22:763

20. Li H, Chen C, Cao X (2015) Essential oils-oriented chiral esters as potential pesticides: asymmetric syntheses, characterization and bio-evaluation. Ind Crops Prod 76:432-436

21. Pan Y, He X (2004) Improvement of the synthesis of 3,4-methylenedioxy phenylpropenic acid. J Shantou Univ Med Coll 17:149-150

22. Singh IP, Jain SK, Kaur A, Singh S, Kumar R, Garg P, Sharma SS, Arora SK (2010) Synthesis and antileishmaniala ctivity of piperoyl-amino acid conjugates. Eur J Med Chem 45:3439-3445

23. Wang S, Bao L, Wang W, Song D, Wang J, Cao X (2018) Heterocyclic pyrrolizinone and indolizinones derived from natural lactam as potential antifungal agents. Fitoterapia 129:257-266

\section{Publisher's Note}

Springer Nature remains neutral with regard to jurisdictional claims in published maps and institutional affiliations.
Ready to submit your research? Choose BMC and benefit from:

- fast, convenient online submission

- thorough peer review by experienced researchers in your field

- rapid publication on acceptance

- support for research data, including large and complex data types

- gold Open Access which fosters wider collaboration and increased citations

- maximum visibility for your research: over 100M website views per year

At BMC, research is always in progress.

Learn more biomedcentral.com/submissions 